\title{
Atomic beam splitters based on light.
}

C. S. Adams, T. Pfau and J. Mlynek.

\author{
Fakultat fur Physik, \\ Universitat Konstanz, \\ D-7750 Konstanz.
}

\begin{abstract}
.
In this paper we review techniques to coherently split an atomic beam using the dipole force. We discuss the interaction of atoms with normal standing wave light field in the context of atomic beam splitters. The case where the atom enters the standing wave at a small angle such that Bragg diffraction and velocity selective resonances are observed is also considered. An alternative approach to realize a coherent beam splitter based on the optical Stern-Gerlach effect is discussed. Finally we consider the interaction of atoms with a magneto-optical potential formed by a polarization gradient light field and a static magnetic field. We show that the Stern-Gerlach effect and the magneto-optical potential produce a more effective beam splitting into states with high tranverse momentum than diffraction from a normal standing wave.
\end{abstract}




\section{Introduction.}

The wave nature of atoms was observed by diffraction from a surface by Stern in 1929 [1]. This was the first experimental demonstation of a beam splitter for atoms. Despite these early experiments using atoms, the field of matter wave optics using electrons and neutrons developed more rapidly. The wave properties of electrons were easier to exploit due to their charge and light mass (hence much larger de Broglie wavelength), and neutrons had the advantage that dynamic Bragg diffraction in pure crystals could be used to realize efficient beam splitters. Two main developments in recent years have lead to a re-newed interest and a rapid growth in the field of atom optics [2]. Firstly improvements in micro-fabrication techniques have made it possible to produce transmission structures with sufficiently fine detail to diffract atomic beams. Secondly the development of intense tunable narrow linewidth lasers has enabled the manipulation of atoms using resonant light forces. These developments can be used to categorise the two approaches to realize optical elements for atoms; diffraction from microfabricated structures and refraction induced by the interaction between the atomic internal degrees of freedom and electromagnetic fields [3].

Beams splitters are a key component in the realization of atom interferometers, could be used as input/output couplers for atom cavities, as state selective elements, or for experiments to study atom correlations (e.g. a Hanbury-BrownTwiss experiment for atoms). In general a beam splitter may have many input and output ports, but here we will restrict the discussion to the simplest and most useful case of a beam splitter with one input and two outputs. The critical parameters of a beam splitter are the splitting angle and in the non-ideal case, the efficiency of the coupling into the two desired output states. A large splitting angle is important for many applications. For example frequently in interferometry the sensitivity is limited by the area enclosed by the two paths of the interferometer. By analogy with optical beam splitters, atomic beam splitters can be divided into polarizing or non-polarizing. As discussed above coherent beam splitters for atoms can be 
realized using diffraction from crystal surfaces [1], diffraction from microfabricated slits and gratings $[4,5,6]$, photon recoil $[7,8]$ and the dipole force [9]. An atomic interferometer based on transmission structures was first demonstrated in a atomic analogue of the Young's double slit experiment [5], and shortly later using a three grating arrangement [6]. Photon recoil beam splitters have also been used to realize atom interferometers using an optical analogue of the Ramsey technique [7,8].

Micro-fabricated structures have some obvious advantages over other techniques. They are passive elements, require little maintainance and can be used with any atomic species. The limitations are that the splitting angles are relatively small compared to those achievable using light forces [10], and the scattering efficiencies to higher orders are low. Also transmission structures necessarily remove a significant percentage of the atomic intensity, ranging from 50\% for a grating to something much higher for a single or double slit.

The second approach to realize optical elements uses light-atom interactions. The deflection of atoms by light can be divided into three categories; the radiation pressure force, single photon recoil and the dipole force. The radiation pressure force involves spontaneous emission which is a stochastic process. Spontaneous decay gives a random momentum kick to the atom and therefore introduces a random phase shift in the wavefunction which destroys the coherence. Thus to form coherent optical components for atoms, it is necessary to avoid spontaneous emission. However as the spontaneous force is dissipative, it opens up the extremely useful possibility of beam conditioning using laser cooling techniques [11], to increase the spatial and spectral brightness and tune the de Broglie wavelength of the beam.

A central question for coherent atom optics is how to avoid spontaneous emission. Obviously this can be achieved by making the interaction time shorter than the spontaneous lifetime. In this respect it can help to use atoms with a long excited state lifetime. An example is the atom interferometer built by Riehle et al. [7]. 
They use calcium atoms where the upper state lifetime is $0.4 \mathrm{~ms}$. The interferometer is based on photon recoil beam splitters, where a travelling wave laser beam is used to excite the atom into a coherent superposition of the ground and excited states by partially absorbing one photon. The excited state component carries the additional momentum of the photon, i.e. the ground and excited state components are split in momentum space and thus become spatially separated in the far-field.

An elegant solution to the problem of spontaneous emission in atom optics has been demonstrated by Kasevich et al. [12]. They effectively eliminate the possibility of spontaneous emission by using stimulated Raman transitions between the two hyperfine levels in the ground state. Using this technique they have demonstrated an interferometer based on slow atoms with an extremely high sensitivity [8].

The dipole force is a coherent process involving the re-distribution of photons through absorption and stimulated emission cycles. The interaction of atoms with a standing wave light field in the absence of spontaneous emission is an example of the application of dipole forces to realize a coherent beam splitter for atoms. In this article we review various techniques to split an atomic beam using the dipole force.

\section{Interaction of atoms with a standing wave light field.}

The diffraction of electrons from a standing wave light field was predicted by Kapitza and Dirac in 1933 [13]. However due to the weakness of the interaction, the effect has not been observed experimentally. The Kapitza-Dirac effect for atoms can be much larger because of the possibility of tuning the light frequency to an internal resonance of the atom. The diffraction of atoms from a standing wave light field is particularly interesting both in the context of our general understanding of light-atom interactions, and the possible applications as coherent beam splitters. The diffraction process can be described either in terms of discrete 
momentum transfer caused by the scattering of photons, or by refraction from the phase grating formed by an optical potential. In the absence of spontaneous emission and for the laser frequency detuned slightly from the atomic resonance, the final momentum distribution consists of discrete peaks separated by two photon momenta, $2 \hbar k$. The first experimental observation of the transfer of individual photon momenta in a standing wave was reported by Moskowitz et al. in 1983 [9].

If the atom decays spontaneously, it receives a momentum kick of $\hbar k$ in a random direction. After many spontaneous emissions the diffraction pattern is destroyed. The transition from coherent to incoherent scattering by a standing wave i.e. the transition from diffraction to diffusion, has been treated theoretically by Tanguy et $a l$. [14] and was demonstrated experimentally by Gould et al. [15].

For a two level atom if each momentum peak is considered as one order of the diffraction pattem, then the intensity $P_{n}$ of order $n$, is given by a Bessel function of order $n$, with an argument that is proportional to the Rabi frequency $\omega_{R}$, i.e. $P_{n}(\tau)=J_{n}^{2}(\pi N)$ where $N$ is the number of Rabi oscillations [16]. The momentum distribution produced by diffraction from a resonant standing wave for $N=10$ is plotted in Figure 1. The total width of the diffraction pattern is proportional to the field amplitude. The same result is given by considering the dressed states of the atom-laser interaction. The eigenvalues of the dressed states can be interpreted as optical potentials and the dipole force is given by the gradient of the dressed state energy [17]. In this case the final momentum distribution is given by the Fourie $r$ transform of

$$
\exp \left[i \int \lambda_{ \pm}(z, t) d t\right]
$$

where $\lambda_{ \pm}$are the energy eigenvalues of the dressed states and $z$ is the propagation direction of the laser beams. In the simplest case of zero laser detuning, the eigenvalues reduce to a sine-wave and the Fourier transform gives the Bessel function solution described above. Note that for exact resonance the atom has an equal probability of emerging from the interaction in the ground or excited state, 
therefore the diffraction pattern is a coherent superposition of diffraction from the two dressed states and the peaks are expected at intervals of $\hbar k$.

Finally we consider the application of the diffraction of atoms from a standing wave laser field as a coherent beam splitter. For low laser intensities, standing wave diffraction for non-zero detuning produces efficient scattering into states with $\pm 2 \hbar k$. However at higher laser intensities (or long interaction times) there is broad spread in momentum space as shown in Figure 1, and the effect can no longer be considered as an efficient beam splitter. For this reason it is necessary to consider other techniques to realize an efficient beam splitter with a large splitting angle.

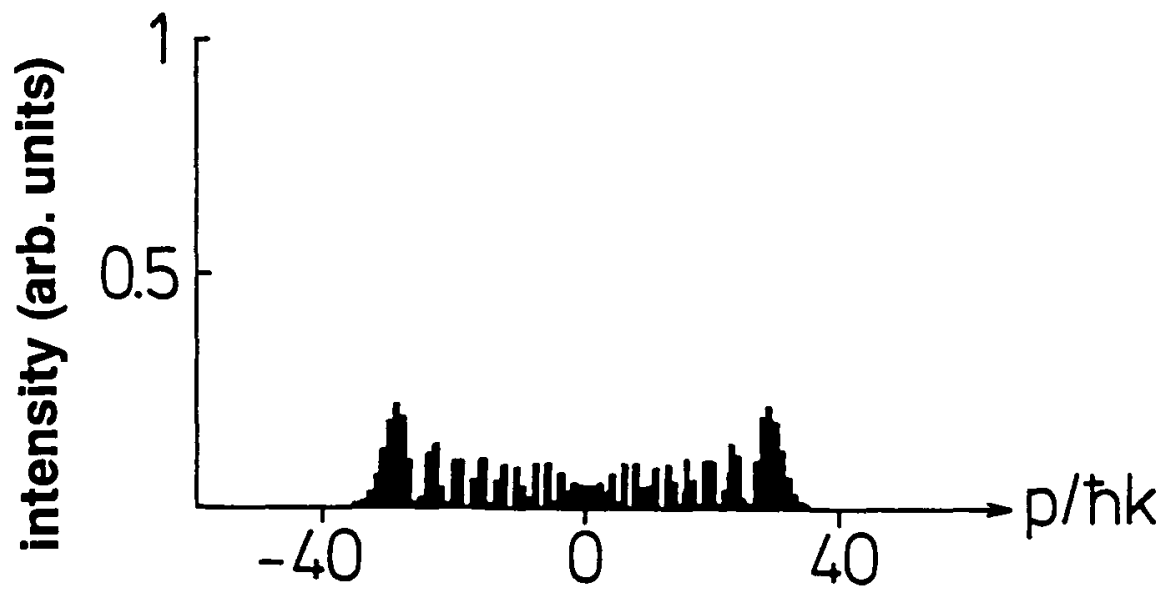

Figure 1. The atomic momentum distribution produced by diffraction of a 2-level atom from a resonant standing wave. 


\section{Bragg diffraction and Doppleron resonances.}

In the above discussion it is assumed that the wave fronts of the standing wave were parallel to the initial momentum of the atom. If we relax this assumption and allow the atom to have a non-zero transverse momentum, other diffraction processes become possible. Two such processes are known as Bragg diffraction and velocity selective or Doppleron resonances.

In the Bragg regime energy and momentum conservation limit the diffraction orders to well defined values. If an atom enters the standing wave field with a transverse momentum $+m \hbar k$, it is efficiently coupled to the state with momentum $-m \hbar k$. For this reason Bragg diffraction could be an effective technique for realizing a 50:50 beam splitter. However the atom undergoes Pendellösung type oscillations between the + and - momentum states with a frequency propotional to the ratio of the Rabi frequency over the detuning. Thus there is a conflict between the detuning required to avoid spontaneous emission and the time required to achieve significant population transfer. A second problem is that as the atomic wavelength is typical much smaller than the optical wavelength the splitter angles are limited to a few photon momentum. First and second order Bragg diffraction and Pendellösung type oscillations have been demonstrated experimentally by Martin et al. [18]. They achieved a coupling efficiency of $45 \%$ for first order Bragg scattering (splitting of $2 \hbar k$ ) and $16 \%$ for second order (splitting of $4 \hbar k$ ).

The Doppleron or velocity selective resonance is a multi-photon process in which the accummulated Doppler shift from absorption and emission processes is compensated by the atom - laser detuning. The application of velocity tuned resonaces as an atomic beam splitter has been proposed [19]. The advantage of the velocity tuned resonace over Bragg diffraction is that the time scale for the process if much faster than the characteristic time of the Pendellösung oscillations. The effect of spontaneous emission on Bragg and velocity tuned resonances has been treated theoretically by Schumacher $e t$ al. [20]. 


\section{Optical Stern Gerlach effect.}

An alternative method to coherently split an atomic beam is based on the state selectiveness of the optical potential. The energy eigenvalues of the two eigenstates in the coupled atom-light system (dressed atoms) differ only by their sign. Therefore a light intensity gradient selects the two eigenstates by acting on them with opposite forces. For an effective beamsplitter a linear field gradient, and an atom in a superposition state of the eigenstates is required. The result is a state selective, i.e. polarizing beamsplitter. The effect is called the optical Stern Gerlach effect because of the strong analogy to the case of a static magnetic moment in a magnetic field gradient and was first proposed by A.P. Kazantsev [21].

Exactly on the atomic resonance the atomic ground state is a 50/50 superposition of the two dressed states in the interaction region. The two outgoing internal states of the atom are orthogonal and both contain an excited state component. If the excited state life time is shorter than the time of flight to the detector, this part will undergo spontaneous emission leading to a partial destruction of the coherence. This causes significant losses in the visibility of an interference pattern if this beam splitter is used in an atom interferometer, on the other hand it opens a direct way to investigate the influence of spontaneous emission on the atomic coherence in a systematic way.

For the case of small detunings from the atomic resonance the ground state is split into unequal fractions of the dressed states by non-adiabatic transitions. As a consequence the splitting ratio gives a direct measure of the non-adiabatic transition rates as a function of detuning. 
In the experimental demonstration of the optical Stern-Gerlach effect [22], the atomic beam was restricted to a region close to a node of a large period standing wave, where there is a nearly linear gradient in the optical potential. The spontaneous emission during the interaction was avoided by using an interaction time much shorter than the excited state lifetime. Using this technique a splitting angle of $\pm 4 \hbar k$ was achieved for the case of metastable helium; however this angle was only restricted by the size of the potential gradient, i.e. by the laser power and the period of the standing wave.

\section{Magneto-optical beam splitter.}

In a normal standing wave the probability for a re-distribution of photons (i.e. absorption from the left and emission to the right or vice versa) is a sinusoidal function of position. This is equivalent to the statement that the force is a sinusoidal function of position. Thus all momentum states between the limits set by the total number of processes are populated with an envelope described by the Bessel function mentioned above. A more efficicient beam splitting effect would occur if the re-distribution of photons, i.e. the force, were a square wave function of position. This would mean that once the atom has absorbed a photon from one direction, it always absorbs from that direction and emits into the other. This turns out to be possible for three level atoms interacting with a magneto-optical potential formed by counter-propagating, orthogonal linearly polarized beams and a static magnetic field applied parallel to the laser propagation direction [23].

The proposed arrangement of the laser beams and the magnetic field is shown schematically in Figure 2. Consider a $J=0$ to $J=1$ transition. The atoms enters the interaction region in the ground state, then absorbs a photon which establishes a linear superposition of the $m_{J}= \pm 1$ states known as an alignment. Absorption of a photon from the other beam (orthogonal polarization) induces the orthogonal alignment. The applied magnetic field causes a precession of the alignment. After one-half of the precession period, the alignment becomes parallel to the orthogonal 
polarization making it more probable for stimulated emission to occur into the other beam. By matching the magnetic precession with rate of the absorption and stimulated emission processes it is possible to arrange that the atom repeats cycles of emission from one direction and emission into the other or vice versa. This process leads to a large, clearly two-peaked splitting of the atomic wavefunction in momentum space.
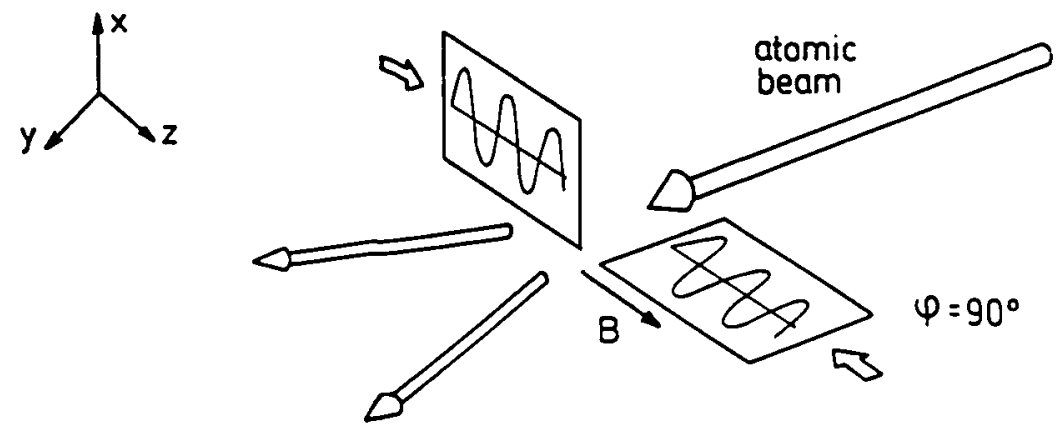

Figure 2. Configuration of laser beams and magnetic field for a magneto-optical beam splitter.

An alternative description is provided by the dressed atom picture. For a particular ratio of the laser intensity and magnetic field strength, one of the eigenstates of the interaction experiences an approximately triangular potential. For a three level atom with a $\mathrm{V}$ level scheme, under experimentally realistic conditions, the ground state evolves adiabatically into the split eigenstate and then back to the ground state. Thus in the case of coherent diffraction, and if the transverse motion of the atom through the potential is negligible (this corresponds to the so called RamanNath regime where the kinetic energy term is omitted from the interaction Hamiltonian), the magneto-optical interaction produces a beam splitting. The 
physical mechanism of the beam splitting effect is similar to the magneto-optical force proposed and demonstrated by Grimm et al. [24].

The width of the two peaks in momentum space is essentially given by the diffraction from the discrete parts of the linear gradients. The width of these regions is $\lambda / 4$, therefore the width of the two peaks in momentum space is $4 \hbar k$ and is independent of the absolute splitting, i.e. the magnitude of the gradient. Therefore this magneto-optical technique is particularly well suited to split the atomic wavefunction into high order momentum states without producing significant broadening of the output beams. As an example a splitting of $\pm 40 \hbar k$ was predicted for metastable helium under experimentally realistic conditions [23]. The expected momentum distribution is shown in Figure 3. This result can be directly compared with the standing wave diffraction pattern shown in Figure 1.

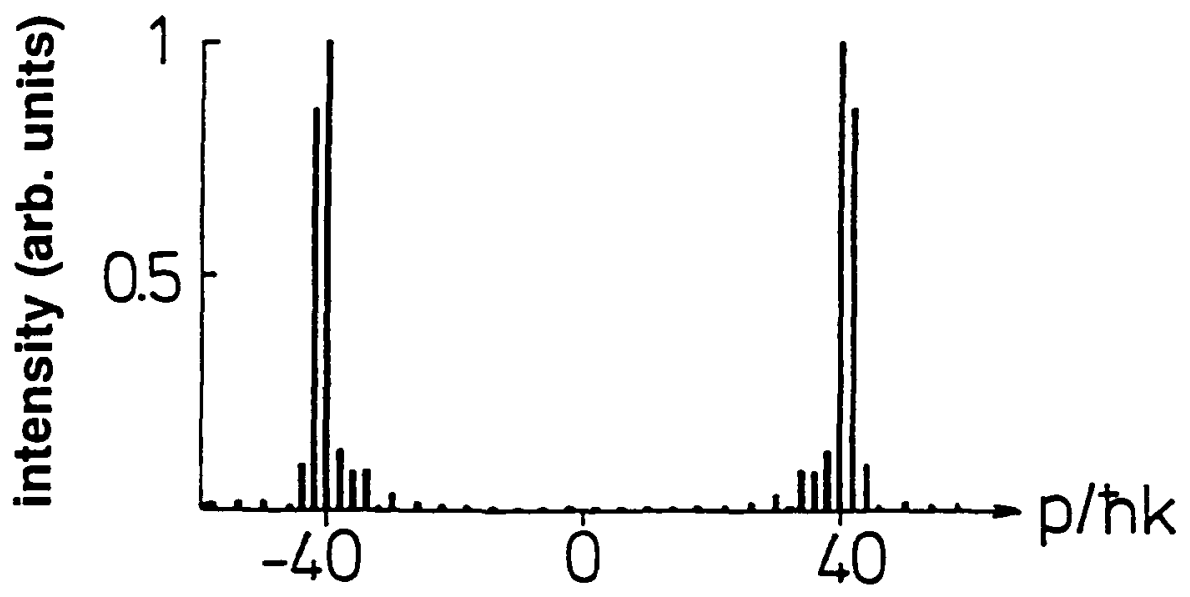

Figure 3. The atomic momentum distribution for a 3-level atom diffracted by a magneto-optical grating. 


\section{Conclusion.}

In this article we have discussed techniques to coherently split an atomic beam using the dipole force. It was shown that diffraction of atoms from a normal standing wave light field produces efficient coupling between low order momentum states, but that the diffraction efficiencies to high transverse momentum states are low. Two techniques for improving the splitting angle and the efficiency of dipole force beam splitters were presented. Firstly the optical Stern-Gerlach effect and secondly diffraction of atoms from a magneto-optical grating. In both these processes the momentum transfer between the atom and the light field is optimised. Thus the Stern-Gerlach and magneto-optical beam splitters produce efficient coupling to high transverse momentum states.

\section{References.}

1. O. Stern, Naturwissensch. 17, 391 (1929), I. Estermann and O. Stern, Z. Phys. 61, 95 (1930).

2. For a recent overview see Optics and interferometry with atoms, ed. by $\mathbf{J}$. Mlynek, V. Balykin and P. Meystre, Special Issue, Appl. Phys. B 54, 321-485 (1992).

3. The terms diffractive and refractive are convenient to distinguish between components which remove part or parts of the wave-front and those which induce a position dependent phase shift. This distinction is not always clear-cut as in the case of interaction of atoms with a resonant standing light wave. The standing wave acts as a phase grating and is a purely refractive element, however it is usual to describe the interaction as diffraction.

4. D. W. Keith, M. L. Schattenburg, H. I. Smith and D. E. Pritchard, Phys. Rev. Lett. 61, 1580 (1988).

5. O. Carnal and J. Mlynek, Phys. Rev. Lett. 66, 2689 (1991). 
6. D.W.Keith, C.R.Ekstrom, Q.A.Turchette and D.E.Pritchard, Phys. Rev. Lett. 66, 2693 (1991).

7. F.Riehle, Th.Kisters, A.Witte, J.Helmcke and C.J.Bordé, Phys. Rev. Lett. 67, 177 (1991).

8. M.Kasevich and S.Chu, Phys. Rev. Lett. 67, 181 (1991).

9. P. E. Moskowitz, P. L. Gould, S. R. Atlas and D. E. Pritchard, Phys. Rev. Lett. 51, 370 (1983).

10. For example consider the diffraction of a supersonic metastable helium beam at $295 \mathrm{~K}$ from a $500 \mathrm{~nm}$ period grating. The first order diffraction angle is approximately $100 \mu \mathrm{rad}$. For comparison a single photon recoil from a light field resonant with the $2^{3} S$ to $3^{3} P$ transition induces a deflection of $50 \mu \mathrm{m}$.

11. For an overview see Laser cooling and trapping of atoms, ed. by S. Chu and C. Wieman, Special Issue, J. Opt. Soc. Am. B6, (1989).

12. M.Kasevich D. S. Weiss, E. Riis, K. Moler, S. Kasapi and S.Chu, Phys. Rev. Lett. 66, 2297 (1991).

13. P. L. Kapitza and P. A. M. Dirac, Proc. Cambridge Philos. Soc. 29, 297 (1933).

14. C. Tanguy, S. Reynaud, M. Matsuoka and C. Cohen-Tannoudji, Opt. Commun. 44, 249 (1983).

15. P.L.Gould, P.J.Martin, G.A.Ruff, R.E.Stoner, J.-L.Picque and D.E.Pritchard, Phys. Rev. A 43, 585 (1991).

16. R.J.Cook and A.F.Bernhardt, Phys. Rev. A18, 2533 (1978).

17. J. Dalibard and C. Cohen-Tannoudji, J. Opt. Soc. Am. B2, 1707 (1985).

18. P.J. Martin, B. G. Oldaker, A. H. Miklich, and D. E. Pritchard, Phys. Rev. Lett., 60, 515 (1988).

19. S. Glasgow, P. Meystre, M. Wilkens and E. M. Wright, Phys. Rev. A43, 2455 (1991).

20. E. Schumacher, M. Wilkens, P. Meystre and S. Glasgow, Appl. Phys. B54, 451 (1992).

21. A.P. Kazantsev, Sov. Phys. JETP 40, 825 (1974).

22. T.Sleator, T.Pfau, V.Balykin, O.Carnal and J.Mlynek, Phys. Rev. Lett. 68, 1996 (1992). 
23. T.Pfau, C.S. Adams, J.Mlynek, "Proposal for a magneto-optical beamsplitter for atoms", submitted to Europhys. Lett. (1992).

24. R.Grimm, Yu.B.Ovchinnikov, A.I.Sidorov and V.S.Letokhov, Opt. Commun. 84, 18 (1991) and R.Grimm, V.S.Letokhov, Yu.B.Ovchinnikov and A.I.Sidorov, JETP Lett. 54, 615 (1991). 